\title{
Neural Mechanisms of Intimate Partner Aggression
}

In press at Biological Psychology

David S. Chester ${ }^{1 *}$, Alexandra M. Martelli ${ }^{1}$, Samuel J. West ${ }^{1}$, Emily N. Lasko ${ }^{1}$, Phoebe Brosnan², Anastasia Makhanova $^{3}$, Andrea L. Meltzer ${ }^{4}$, James K. McNulty ${ }^{4}$

${ }^{1}$ Department of Psychology, Virginia Commonwealth University, Richmond, VA 23284

2Department of Psychological \& Brain Sciences, Boston University, Boston, MA 02215

${ }^{3}$ Department of Psychological Science, University of Arkansas, Fayetteville, AR 72701

${ }^{4}$ Department of Psychology, Florida State University, Tallahassee, FL 32306

${ }^{*}$ Corresponding Author:

David S. Chester, Department of Psychology, Virginia Commonwealth University, 806

West Franklin St., Richmond, VA, 23284, 1-804-828-7624, dschester@vcu.edu 


\begin{abstract}
People sometimes hurt those they profess to love; yet our understanding of intimate partner aggression (IPA) and its causes remains incomplete. We examined brain activity using functional magnetic resonance imaging (fMRI) in an ethnically and racially diverse sample of 50 female-male, monogamous romantic couples as they completed an aggression task against their intimate partner, a close friend, and a different-sex stranger. Laboratory and real-world IPA were uniquely associated with altered activity within and connectivity between cortical midline structures that subserve social cognition and the computation of value. Men's IPA most corresponded to lower posterior cingulate reactivity during provocation and women's IPA most corresponded to lower ventromedial prefrontal cortex activity during IPA itself. Actor-partner independence modeling suggested women's IPA may correspond to their male partner's neural reactivity to provocation. Broadly, these findings highlight the importance of self-regulatory functions of the medial cortex and away from effortful inhibition subserved by dorsolateral cortices.
\end{abstract}

Keywords: intimate partner aggression, intimate partner violence, fMRI, prefrontal cortex, implicit partner attitudes 


\section{Highlights}

- First functional brain imaging study during intimate partner aggression (IPA)

- IPA linked to blunted cortical midline activity during provocation and aggression

- IPA linked to greater connectivity between ventral and dorsal MPFC

- Men's IPA linked more to neural response to provocation

- Women's IPA linked more to neural response during aggression itself 


\section{Introduction}

Despite millennia of cultural advancement, humans still show a remarkable propensity for aggression towards even their closest connections - their intimate partners (Black et al., 2011). Researchers have amassed a voluminous literature on intimate partner aggression (IPA), focusing mostly on the sociological and psychological predictors and consequences of IPA perpetration and victimization (Capaldi, Knoble, Shortt, \& Kim, 2012; Chester \& DeWall, 2017). Yet given that all behavior is ultimately based in the brain, a comprehensive understanding of IPA requires knowing its neurobiological bases. Though multiple studies have examined the neural correlates of aggression perpetrated against strangers (Chester \& DeWall, 2016; Krämer, Jansma, Tempelmann, \& Münte, 2007), neuroimaging research into IPA is a relatively new and growing literature. The present research represents an initial snapshot of human brain activity that occurred during IPA, which provides new insights into its neural and psychological bases.

\section{Current Models of IPA}

Aggression is defined as any attempt to harm someone else against their will (Allen \& Anderson, 2017). It can range in the severity of the harm intended from mild to moderate to extreme. At this extreme of harm-doing, aggression becomes violence. IPA refers to deliberate attempts to harm one's intimate partner against their will, ranging across all forms of severity (Chester \& DeWall, 2017). Accordingly, intimate partner violence refers to extremely harmful acts of IPA. Across all of its forms, IPA is remarkably costly. Approximately one-third of women and one-fourth of men have been victims of intimate partner violence at some point in their lives (Black et al., 2011). The 
immense toll of violent and less severe forms of IPA on victims and society necessitates research into the forces that cause and constrain the perpetration of such acts - from the most severe to the least.

IPA most clearly differs from other forms of aggression in its target (i.e., intimate partners versus people with whom perpetrators are not intimate), but there are key similarities between IPA and aggression that is not directed at intimate partners. Both forms of aggression can be reactive (i.e., angry and impulsive retaliation in response to perceived provocation) and proactive (i.e., premeditated and 'cold' in service of a broader goal) in function (Halmos, Parrott, Henrich, \& Eckhardt, 2020). They can also both be overt (i.e., physical and direct) and relational (i.e., verbal and indirect) in form. That said, correlations between intimate partner and general aggression are only moderate in magnitude (e.g., Thornton, Graham-Kevan, \& Archer, 2013), suggesting they are distinct behavioral phenotypes. Yet psychological variables that accurately differentiate these two forms of aggression remain largely undiscovered.

To date, most IPA research has focused on the large-scale societal and proximal psychological forces that promote it (Capaldi et al., 2012; Chester \& DeWall, 2017; Finkel et al., 2012). Although these findings are crucial for developing an understanding of IPA, they are limited in several ways. First, sociological studies of macro-societal predictors (e.g., socioeconomic status) and historical events (e.g., parental IPA) are less able to inform proximal-level treatments, interventions, and prevention efforts. Second, sociological and experimental investigations often rely on self-reports and partnerreports, the validity of which can be undermined by psychological biases (e.g., Bell \& Naugle, 2007). Third, such research is largely silent on the underlying biological 
processes that shape IPA. In contrast, neuroscience has advanced our understanding of the biological processes that underlie many social behaviors and this approach is often able to identify undiscovered mechanisms and circumvent biases in self-reports.

\section{A Biological Substrate of IPA: The Medial Prefrontal Cortex}

Aggression has clear biological bases in brain function and structure.

Specifically, the medial prefrontal cortex (MPFC) has been particularly implicated as a neural substrate of aggressive behavior. The MPFC, which spans a large swath of neocortex behind the forehead, exhibits a dorsal-to-ventral functional and spatial gradient. Indeed, this gradient in MPFC activity has been linked to aggression against strangers across multiple studies. Although several studies have found a positive correlation between ventral MPFC activity and aggression (Buades-Rotger, Beyer, \& Krämer, 2017; Repple et al., 2017), the majority of neuroimaging studies have shown a correspondence between ventral MPFC activity with lower aggression and dorsal MPFC activity with greater aggression (Beyer, Münte, Göttlich, \& Krämer, 2015; Chester, Lynam, Milich, \& DeWall, 2017; Denson, Pedersen, Ronquillo, \& Nandy, 2009; Krämer et al., 2007; Lotze, Veit, Anders \& Birbaumer, 2007).

The MPFC has many functions, but it subserves two psychological processes that may be particularly relevant to IPA: self-partner overlap and partner value. Indeed, the most comprehensive meta-analysis to date highlighted that among the MPFC's various functions, the psychological processes with the strongest evidence for representation in the MPFC included self/social cognitions (e.g., self-other overlap) and value computations (Lieberman, Straccia, Meyer, Du, \& Tan, 2019). Given the wellestablished role of these two psychological processes for relationship functioning (Aron, 
Aron, Tudor, \& Nelson, 1991; Kelley \& Thibaut, 1978), we focused our investigation on self-partner overlap and partner value.

Self-partner overlap. A core finding of social-cognitive neuroscience is that the MPFC differentiates among the self, familiar others, and unfamiliar others (Denny, Kober, Wager, \& Ochsner, 2012). Indeed, more ventral regions of the MPFC encode stimuli and judgments that are relevant to the self or close others, whereas such processes are encoded in increasing dorsal aspects of the MPFC for more unfamiliar and distant social targets (Mitchell, Macrae, \& Banaji, 2006). As such, the psychological overlap between representations of the self and one's intimate partner can be indexed by the extent to which the partner recruits relatively ventral MPFC (VMPFC) functions, as opposed to dorsal MPFC (DMPFC) activity (Kawamichi et al., 2013). The extent to which people encode their intimate partner as overlapping with their own self-concept is a critical factor in relationship outcomes (Aron et al., 1991). Most notably, such mental representations of self-other overlap are crucial in promoting prosocial behavior and constraining antisocial tendencies (Galinsky, Ku, \& Wang, 2005). It is thus likely that self-partner overlap that is subserved by the VMPFC would constrain IPA.

Partner value. The VMPFC may constrain IPA because it is a core hub of the brain's reward network and, in this role, it serves to compute the subjective value of actions, goals, and objects (Liu, Hairston, Schrier, \& Fan, 2011). This value function allows for the creation of self-other overlap, as the self and close others are represented as intrinsically positive and valuable psychological constructs (Chavez, Heatherton, \& Wagner, 2016). Indeed, the VMPFC appears to simultaneously encode the extent to which intimate partners overlap with the self as well as their intrinsic value (Hughes \& 
Beer, 2012). Notably, perceived partner value is an important psychological factor in the context of IPA. Classic relationships theories such as interdependence theory (Kelley \& Thibaut, 1978), suggest that relationship outcomes are highly contingent on the perception that one's partner possesses value relative to other potential mates. Such perceptions of value inhibit IPA (Figueredo et al., 2018). As such, there is reason to expect that greater implicit partner value, as encoded in the VMPFC, is a likely mechanism underlying reduced IPA.

From a social cognitive perspective, the extent to which one values a given target is conceptualized as an attitude - defined as the evaluative experience activated by a target (Fazio, 2007). People are strongly motivated to perceive value in a romantic partner, which biases such self-reports (Murray, 1999). Thus, implicit assessments are often better indicators of subjective value and more predictive of relationship-relevant outcomes (LeBel \& Campbell, 2009; McNulty, Olson, Meltzer, \& Shaffer, 2013).

\section{The Neuroscience of IPA Perpetration}

The study of the brain bases of IPA perpetration is a burgeoning and young field, but several key findings have emerged that largely center on the MPFC. Men who were convicted of perpetrating severe IPA, compared to men who were convicted of other crimes, exhibited thinner cortical structure in the MPFC and posterior cingulate cortex (Verdejo-Román, Bueso-Izquierdo, Daugherty, Pérez-García, \& Hidalgo-Ruzzante, 2019). Further, such IPA perpetrators exhibited blunted default mode network (including the VMPFC) activity when deciding whether to perpetrate IPA in a hypothetical scenario (Marín-Morales et al., 2020). These findings suggest that the MPFC is a place of convergence for the young neuroscience of IPA perpetration. 
There is further evidence that links IPA with a functional bias in MPFC activity, away from the VMPFC and towards the DMPFC. For example, offenders who were convicted of IPA, compared to offenders who were convicted of other kinds of offenses, exhibited greater DMPFC reactivity to images of IPA (Bueso-Izquierdo et al., 2016). Likewise, non-incarcerated IPA perpetrators exhibited bias toward DMPFC reactivity to interpersonal provocation (Chester \& DeWall, 2019). In another study, even listening to audio recordings of an argument with one's partner was enough to recruit DMPFC activity (Flanagan et al., 2019). However, these studies did not examine neural activity during acts of IPA or the psychological processes that may explain this association.

\section{The Present Study}

To fill these gaps in the literature, we conducted a functional neuroimaging study in which both partners within an intimate couple completed a validated aggression paradigm against each other and two control targets (a close friend, a different-sex stranger) while undergoing functional magnetic resonance imaging (fMRI) of the brain. For ethical reasons, this MRI aggression task was not designed to capture severe, intimate partner violence. Instead, this measure captured a less severe, moderate level of aggression. Yet, such scores obtained from laboratory aggression paradigms do exhibit external validity in the form of positive correlations with measures of real-world violence (King \& Russell, 2019). Therefore, this MRI aggression task was intended to capture moderate IPA within the ethical constraints of the laboratory, while still shedding light on neural mechanisms that might play a role in more severe acts of IPA. To gain further insight into these more violent forms of IPA, we also included a self-report measure of overt, physical acts of perpetrating intimate partner violence. These data 
were used to test the preregistered predictions that lower VMPFC activity and greater DMPFC during an aggression decision would be associated with greater IPA.

Alongside these measures of aggression and violence, participants completed several measures to identify the psychological processes represented by altered activity in the VMPFC and DMPFC. These reverse inference measures included an implicit measure of how much participants valued their partner, as well as self-report measures of explicit partner value and perceived self-partner overlap. Using these measures, we predicted that greater self-partner overlap and implicitly-assessed partner value would be reflected in greater IPA-related activity in VMPFC and less IPA-related activity in DMPFC. These predictions are depicted in our conceptual model (Figure 1).

\section{Figure 1}

Conceptual Model of the Present Research

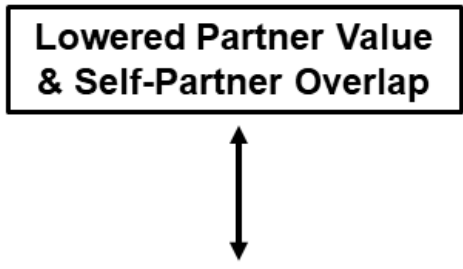

Greater DMPFC Activity \& Lesser VMPFC Activity
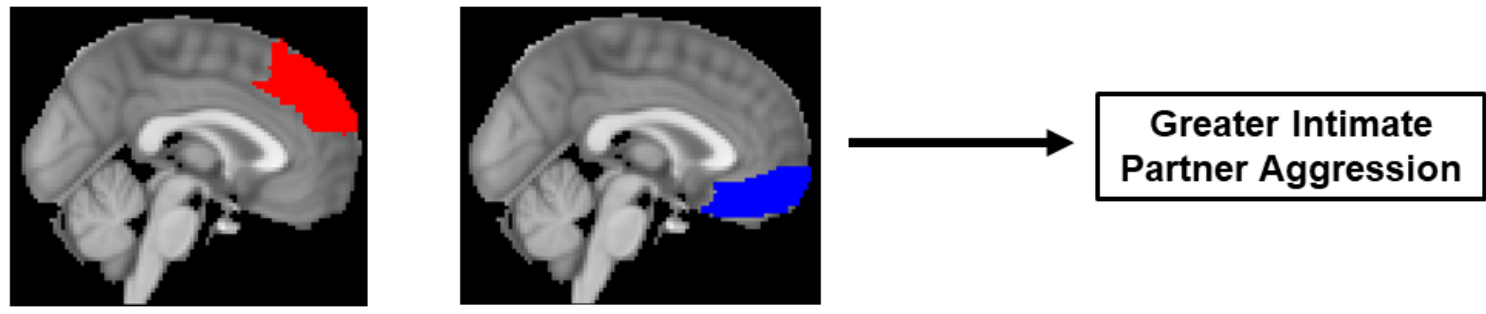

Intimate Partner Aggression Decision

Intimate Partner Aggression Outcome

\section{Methods}

\section{Open Practices Statement}

The preregistration plan for the present research is publicly available 
(https://osf.io/un2fy). Because we collected extremely sensitive data from both members of romantic couples, the potential likelihood for a confidentiality breach and the impact such a breach could have is too high to publicly share the raw data from this study. As recommended by Finkel, Eastwick, and Reis (2015), we will share such de-identified data with competent professionals who request it from the corresponding author.

\section{Ethical Considerations Statement}

Research activities summarized in the manuscript were pre-approved by an institutional review board and carried out in accordance with institutional and national regulations.

\section{Statistical Power Statement}

Statistical power was determined by logistical constraints (i.e., data collection continued until available funds were exhausted) and not an a priori power analysis. We preregistered a planned sample size of $N=50$ individuals ( 25 couples), but we increased our sample size to $N=100$ individuals (from 51 couples) in order to allow our analyses $80 \%$ power at $\alpha=.05$ to detect zero-order main effects of at least $r=.28$ (an effect size that approximates the average effects reported in the aggression literature; Richard, Bond Jr., \& Stokes-Zoota, 2003).

\section{Participants}

Initial participants were 51 couples ( $N=102$ individuals) recruited from the Richmond VA community ( $n=73$ individuals) and from an introductory psychology subject pool ( $n=29$ individuals). Each couple was composed of one man and one woman who were both heterosexual and monogamous for at least six continuous months. We used an online screening questionnaire, which assessed the following 
additional exclusionary criteria that ensured participant safety and MRI data quality: age outside the range of 18 to 35 , body mass index above 30 , claustrophobia, color blindness, mental or neural pathology, metallic objects in the body, non-right-hand dominance, prior head trauma, and current psychoactive medication use. Despite explicit and repeated instructions not to, two male participants arrived at the study with metallic objects in their body that precluded participation in the study. Thus, our final sample consisted of 100 healthy, right-handed, young adults from 51 couples ( $51 \%$ female, $49 \%$ male; age: $M=21.61, S D=3.73$, range $=18-35$; months in relationship: $M$ $=24.01, S D=14.98$, range $=6-84 ; 4 \%$ dating casually, $83 \%$ dating seriously, $5 \%$ engaged, $5 \%$ married, $3 \%$ missing relationship-type data), with considerable diversity (19\% Hispanic, 78\% Non-Hispanic, 3\% missing ethnicity data; 7\% African-American, $22 \%$ Asian-American, 1\% Native American, 45\% White, 22\% Other, 3\% missing race data). Each participant received either $\$ 100$ or research credit for their participation.

\section{Materials}

MRI aggression task. To assess aggression directed towards participants' intimate partners and two control targets (i.e., a close friend, a different-sex stranger), we adapted the well-established Taylor Aggression Paradigm, which has been effectively implemented in the fMRI environment (Chester \& DeWall, 2016; Krämer et al., 2007). This paradigm exhibits good evidence of external, construct, criterion, and convergent validities (Chester \& Lasko, 2019; Giancola \& Parrott, 2008) and does not elicit excessive distress in participants (Parrott, Miller, \& Hudepohl, 2015). As in previous iterations of the paradigm, participants repeatedly competed against an opponent to see who could press a button faster when a prompt appeared. In reality, 
there was no other person and participants completed the task against a computer program. As an ostensible motivational component of the task, participants were punished if they lost a given round of competition by receiving an aversive noise blast through a pair of headphones at the volume their fictitious opponent set for them. Conversely, if participants won the round of competition, their fictitious opponent ostensibly heard the noise blast at the volume that participants previously set for them and the participant heard nothing. The task was modified to include a within-participants manipulation of aggression target (partner, close friend, different-sex stranger [i.e., male stranger for female participants, female stranger for male participants]).

The aggression task consisted of three blocks of eight trials (24 trials total, 8 per target; Figure 2). Participants completed the task twice, across two separate fMRI runs, for a total of 48 trials, 16 per target. Each block corresponded to a different target (i.e., partner, friend, or stranger) and the order of these blocks was randomized for each participant. 


\section{Figure 2}

An Example of One Block of the MRI Aggression Task

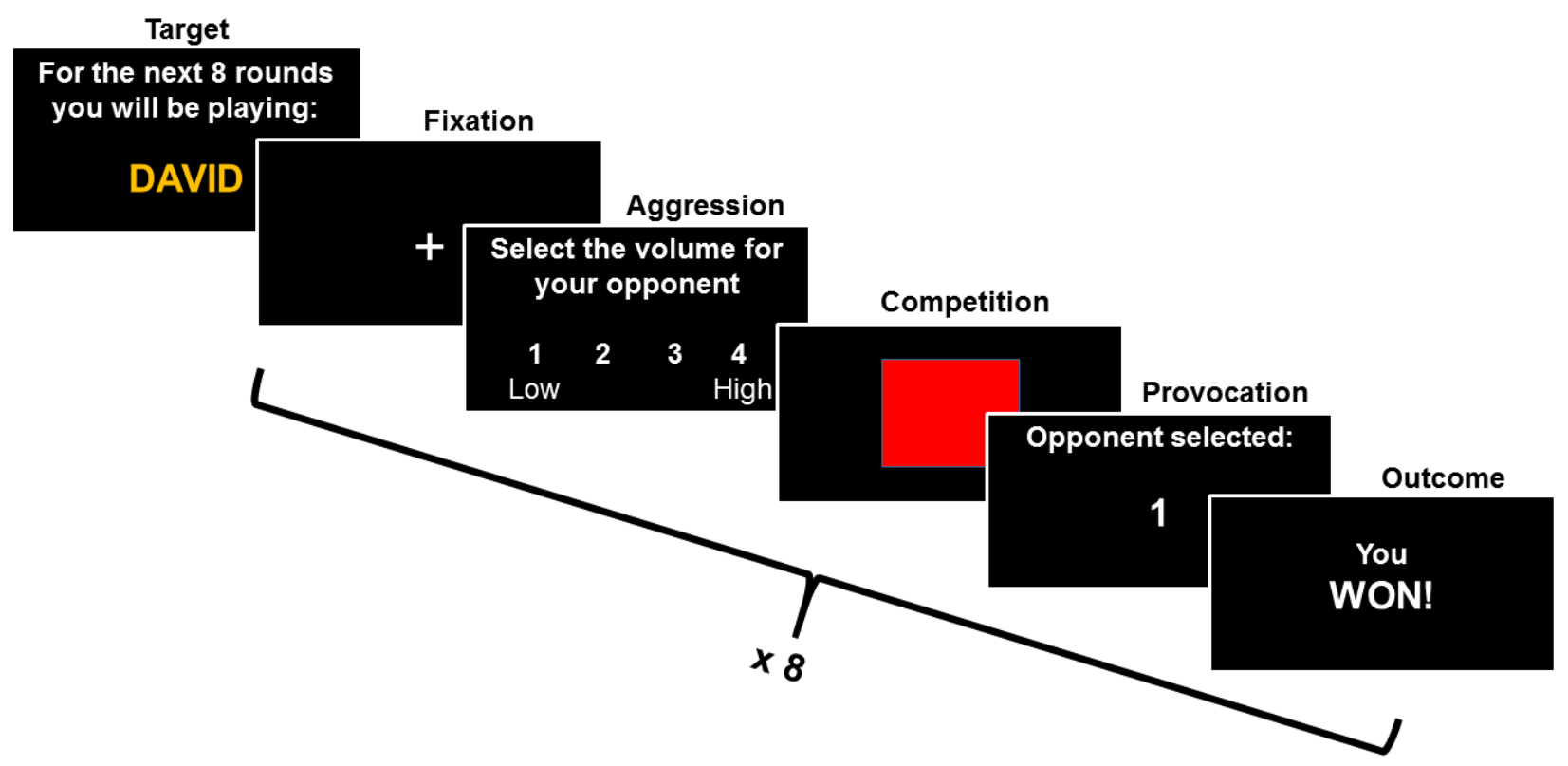

At the beginning of each block, a screen indicated the first name of the participant's opponent (5s duration). Each subsequent trial consisted of six events. First, participants passively viewed a fixation cross that modeled baseline neural activity (randomly jittered between $2.5 \mathrm{~s}$ and $5 \mathrm{~s}$ durations). Then, participants selected the volume of the noise blast to administer to their opponent along a 1 (Low) to 4 (High) scale (5s duration). A blank screen then appeared followed by a red square that indicated to participants that they should press the button as fast as possible to compete against their opponent (5s duration). Participants then saw what volume level their opponent set for them (5s duration). Finally, participants saw whether they won or lost the competition and, if they lost, experienced the corresponding noise blast (2.5s duration). Opponents' volume settings and the outcome of each trial were randomized with replacement, such that any volume and outcome combination could occur on any 
given trial. This randomization approach differed from previous iterations of this aggression task that employed standardized sequences of opponent settings and outcomes. This methodological departure was performed because no research has established an optimal schedule of provocations and outcomes for the task, and therefore we did not want our results to be influenced by arbitrary decisions made in setting these variable components of the task. All participants were asked if they could hear the noise blasts, but we did not inquire as to how aversive they felt. It may be that the earplugs and scanner noise rendered the noise blasts as non-aversive, though higher values selected by participants would still reflect relatively greater levels of the aversive noise. Due to a software error, the first 12 participants could not hear the noise blasts (though they could still see the volume that their opponents had ostensibly selected for them), but they were told that their opponents could still, in fact, hear the blasts - because they likely continued to believe the aggression they inflicted on their opponents was real, we retained these participants in our analyses.

Abuse Within Intimate Relationships Scale. The 26-item AWIRS quantified five forms of IPA (Borjesson, Aarons, \& Dunn, 2003): overt violence, emotional abuse, deception, verbal abuse, and restrictive violence. Participants self-reported the frequency with which they perpetrated each form of IPA from 0 (never) to 7 (more than once a day). Deviating from our preregistration, we focused only on the Overt Violence subscale (seven items; e.g., I have physically attacked my partner) because of the physical nature of aggression (i.e., physical noise blasts) as it was assessed by our fMRI aggression paradigm.

Implicit Partner Attitudes Task. To measure participants' implicit partner 
valuations, we employed an implicit partner attitudes task that examined the speed with which participants associated images of their partners with negative and positive concepts (McNulty et al., 2013). Implicit value was assessed as a combination of (A) how quickly participants implicitly associated their partner with positive concepts and (B) how slowly they implicitly associated them with negative concepts. In this task, participants categorized words as either 1 (Negative) or 2 (Positive), as quickly and accurately as possible. Prior to each categorization trial, participants viewed a fixation cross (1s duration) and then an image of either their intimate partner or an attractive, different-sex stranger for $150 \mathrm{~ms}$ [i.e., male stranger for female participants, female stranger for male participants]. Partner stimuli were one of five images that we asked participants to send to us prior to the study. Stranger stimuli were one of five, differentsex, same-race images acquired from dating websites and pre-rated as being physically attractive by a pilot sample of undergraduate students.

Participants completed 16 practice trials of the task ( 8 negative, 8 positive words), in which the prime images were simply replaced by a row of three asterisks. Participants then completed 32 trials of the actual task that were divided into four conditions (8 trials per condition): partner prime - negative word, partner prime - positive word, stranger prime - negative word, stranger prime - positive word. Response times were averaged across all eight trials within each condition. We computed an implicit 'partner value' index for each participant by subtracting mean response times of the partner prime - positive word condition from the partner prime - negative word condition, such that higher values reflected more positive partner attitudes overall $-\mathrm{a}$ combination of being slower to associate partner primes with negative words and being 
faster to associate partner primes with positive words (McNulty et al., 2013).

Inclusion of Other in the Self Scale. We employed the 1-item IOS scale to operationalize participants' perceptions of closeness between themselves and their intimate partner (Aron, Aron, \& Smollan, 1992). To do so, participants selected one pair of circles from an array of seven pairs of circles that varied in the extent to which they depicted overlap between the two circles (one labeled as 'Self' and the other labeled as 'Other'). The circles were assigned values ranging from 1 (no overlap) to 7 (much overlap).

Perceived Relationship Quality Components inventory. To contrast implicit partner value against explicit value in an exploratory manner, we employed the 18-item PRQC inventory to operationalize participants' explicit perceptions of their partner's value to them (Fletcher, Simpson, \& Thomas, 2000). To do so, participants responded to 18 questions about their current romantic partner that were sub-divided into six threeitem component subscales: commitment, intimacy, love, passion, satisfaction, and trust. Participants' used a response scale ranging from 1 (not at all) to 7 (very much).

\section{Procedure}

Participants were first sent an informational email that instructed both partners from each couple to each identify one unique person who they considered a 'close friend' of any gender (i.e., two close friends per couple). Participants provided the study team with contact information for both close friends, and the study team contacted them, alongside the couple, to schedule an appointment in which the couple would arrive at the MRI laboratory and both close friends would be available to participate via the internet from wherever they chose. Both partners from each couple arrived at the 
MRI center to complete a three-hour laboratory session. Once participants were escorted back into our laboratory, we emailed both of their close friends to inform them that they would not, in fact, be needed to participate in the study. The laboratory session employed a parallel-procedures design, in which each member of a given couple completed the same tasks but in different temporal sequences (Figure 3). Each couple was immediately separated and randomly assigned to complete 'procedure A' or 'procedure B,' in order to stagger participants' MRI scans while still having them believe they were both being simultaneously scanned.

\section{Figure 3}

Schematic of the Study's Parallel Research Procedure

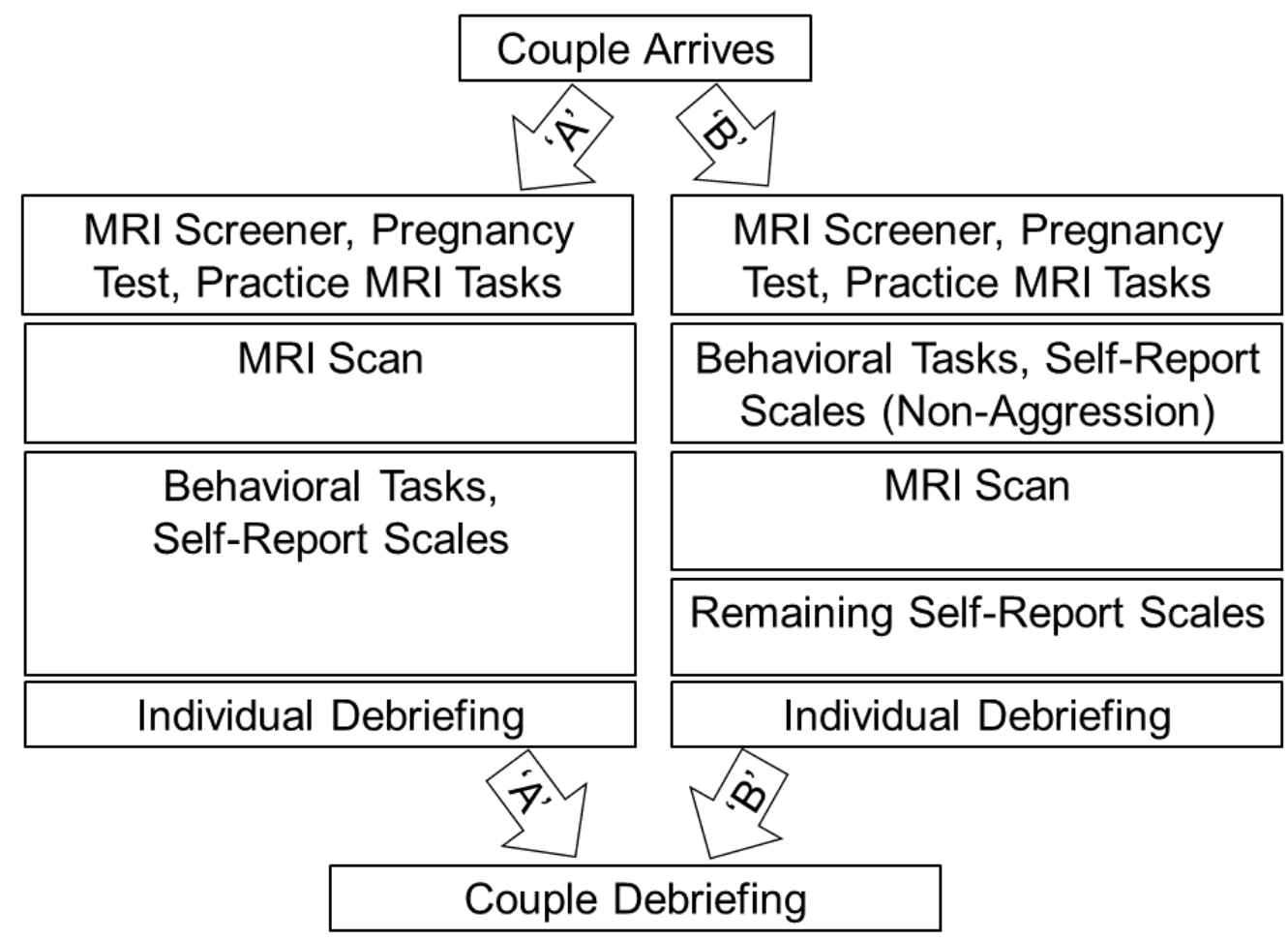

Both participants were again screened for MRI safety, completed a urine pregnancy test (women only), and then practiced the computer tasks they would complete in the MRI scanner. Participant ' $A$ ' then completed the MRI scanning 
procedures while participant ' $\mathrm{B}$ ' completed a battery of behavioral tasks that included the implicit partner attitudes task and self-report questionnaires that did not assess aggression (in order to avoid demand characteristics). Participants then switched, with participant ' $A$ ' returning to complete the battery of behavioral tasks that included the implicit partner attitudes task and self-report measures (of both aggression and nonaggression traits), while participant ' $\mathrm{B}$ ' completed the MRI scanning procedures. After the MRI scan, participant 'B' completed the remaining self-report measures of aggression. At the end of the laboratory session, we gave both participants a twoquestion, funneling suspicion interview to assess their knowledge regarding the deceptive elements of the study. One participant indicated suspicion surrounding their fictitious partners when asked "what did you think this study was about?" and six more participants indicated such suspicion when subsequently asked the leading question "did anything seem strange or unusual to you during this study?" Due to these low suspicion rates and the lack of a preregistered exclusion strategy, we did not exclude these seven participants who indicated suspicion. We then individually debriefed participants as to the deception and true purposes of the study and provided each with mental health resources. Upon confirmation that both participants $(A)$ were not distressed by our procedures, (B) understood that they did not actually interact with their intimate partner on the aggression task, (C) did not feel angry or aggressive towards their partner, and (D) felt safe and comfortable to interact with their partner, the participants were reunited and again debriefed as a couple, compensated, and dismissed with thanks. We acquired a Certificate of Confidentiality (CC-AA-18-025) from the National Institutes of Health to protect participant privacy. 


\section{MRI Data Acquisition}

All MRI data were obtained using a Philips Ingenia 3.0 Tesla scanner with a 32channel headcoil. Echo planar BOLD images were acquired with a T2*-weighted gradient across the entire brain with: a 3D shim, field of view $(F O V)=224 \mathrm{~mm} \times 224 \mathrm{~mm}$, echo time $(T E)=28 \mathrm{~ms}$, repetition time $(T R)=2.5 \mathrm{~s}$, slice thickness $=3.5 \mathrm{~mm}, 40$ interleaved slices, flip angle $=90^{\circ}$. Eight dummy functional volumes were acquired and discarded from the beginning of each functional run to allow for signal equilibration. To allow for registration to native space, a coplanar, magnetization-prepared rapid gradient-echo (MPRAGE) sequence was also acquired from each participant, with: FOV $=240 \mathrm{~mm} \times 259 \mathrm{~mm}$, slice thickness $=1 \mathrm{~mm}, \mathrm{TE}=3.7 \mathrm{~ms}, \mathrm{TR}=8.1 \mathrm{~s}, 160$ sagittal slices, flip angle $=6^{\circ}$.

\section{MRI Data Preprocessing}

The Oxford Center for Functional MRI of the Brain's Software Library (FSL version 6.0) was used to conduct all preprocessing and fMRI analyses (Smith et al., 2004). Reconstructed functional volumes underwent head motion correction to the median functional volume using FSL's MCFLIRT tool. FSL's Brain Extraction Tool was used to remove non-brain tissue from all functional and structural volumes. After a series of data-quality checks, functional volumes underwent interleaved slice-timing correction, pre-whitening, spatial smoothing (using a $5 \mathrm{~mm}$ full-width-half-maximum Gaussian kernel), and temporal high-pass filtering (100s cutoff). These processed brain volumes were then fed into subsequent data analyses.

\section{Deviations From Preregistered Analytic Plan}

The majority of MRI and behavioral data analyses presented in this manuscript 
were deviations from our preregistration plan, due to our later realization that the preregistered analytic plan was inappropriate for reasons that are noted throughout our analyses. As such all analyses should be viewed as exploratory and not confirmatory.

\section{MRI Data Analysis}

Preprocessed fMRI data were analyzed using general linear modeling. Noise blast settings from the MRI aggression task were missing from our first two participants because the task software failed to record their responses. These participants were excluded from all fMRI analyses, resulting in a final sample of $N=98$.

First level (within-participant, within-run). Participants' whole-brain functional volumes from each run of the aggression task were entered into a fixed-effects general linear model (GLM) that modeled events using a canonical double-gamma hemodynamic response function with a temporal derivative. Regressors-of-interest for the aggression task included aggression decision events towards the partner, friend, and stranger (i.e., those in which participants selected the volume for their fictitious opponent) and provocation events from the partner, friend, and stranger (i.e., those in which participants viewed what volume their fictitious opponent selected for them). All other events were included as nuisance regressors and fixation events were left unmodeled to create an implicit functional baseline. Six head motion parameters from each participant were modeled as nuisance regressors.

Provocation events (i.e., in which participants viewed what volume their fictitious opponent selected for them) were modeled from the partner, friend, and stranger in both non-modulated and parametrically modulated forms. In the modulated form, each provocation event was weighted by the 1-4 volume level selected by the computer. 
Such parametric modulation was not performed for aggression decision events (i.e., in which participants selected the volume for their fictitious opponent), as multiple participants did not respond within the 5 -second timeframe, producing missing data.

We originally planned to categorize aggression decision events as 'retaliatory' and 'non-retaliatory' based on whether they were preceded by provocation levels in the 'high' or 'low' range, but we deviated from this plan by modeling provocation in a continuous manner and correlated it with participants' overall IPA. This deviation was motivated by our determination that the preregistered procedure would unnecessarily and arbitrarily categorize a continuous manipulation and thereby unnecessarily complicate our experimental design.

Linear contrasts compared Partner Aggression Decisions > Non-Partner (i.e., Friend + Stranger) Aggression Decisions and Partner Provocation > Non-Partner (i.e., Friend + Stranger) Provocation. Each participant's resulting contrast brain maps from these analyses were first linearly registered to native space structural volumes and then spatially normalized to a Montreal Neurological Institute (MNI) stereotaxic space template image (resampled into $2 \mathrm{~mm}^{3}$ isotropic voxels).

First level (within-participant, across-runs). A fixed-effects GLM then aggregated the contrast maps from each participant's two functional runs into a single, across-run contrast map.

Second level (across-participants). Participants' contrast maps from the second level were then fed into a group level, mixed-effects GLM that created wholebrain group average maps for each contrast. This GLM also served as a whole-brain regression analysis that modeled each participant's aggression towards his or her 
partner. Aggression values were created by averaging participants' volume settings towards their intimate partner across both runs and all eight trials within each run and then mean-centering those aggregated values. Cluster-based, family-wise error correction using Gaussian random field theory was then applied across the entire brain to each of the group activation maps (corrected threshold: $Z>2.3, p<.05$; Heller, Stanley, Yekutieli, Rubin, \& Benjamini, 2006).

Psycho-physiological interaction (PPI) analyses. To assess functional connectivity during Partner > Non-Partner Aggression Decisions, a psychophysiological interaction (PPI) analysis was performed using the significant VMPFC clusters from the group-level, whole-brain analysis as the seed region. To do so, we re-ran the first level (within-participants) GLM with the addition of two new regressors: the mean-centered time course of the seed region, and an interaction term (i.e., the PPI regressor) that multiplied the VMPFC time course by the non-modulated Partner Aggression Decisions regressor at the run-level. A linear contrast compared participants' implicit baseline against this PPI regressor (PPI > Baseline). These PPI maps were then examined across runs and participants in a group-level GLM that modeled each participant's aggression towards his or her partner as a group-level regressor.

\section{Behavioral Analyses}

Repeated-measures ANalysis-Of-VAriance (ANOVA) tests were employed to examine the within-participants' categorical structure of the implicit partner attitudes task using JASP (v. 0.11.1; JASP Team, 2020). To account for the nested design, brainbehavior correlations were assessed using multilevel models in the Ime4 (Bates, Mächler, Bolker, \& Walker, 2014) and ImerTest (v. 3.1.3; Kuznetsova, Brockhoff, \& 
Christensen, 2017) packages for R (v. 3.6.2; R Core Team, 2020), which nested participants within romantic dyads and specified random intercepts.

\section{Actor-Partner Independence Models (APIM)}

APIMs were conducted using structural equation modeling with maximum likelihood estimation via the APIM_SEM online utility (https://apimsem.ugent.be/shiny/apim_sem/; Stas, Kenny, Mayer, \& Loeys, 2018). Each fully-saturated APIM included four variables (i.e., a predictor and outcome variable for each female and male dyad member) and estimated their fixed intercepts and variances, the covariance between the dyad members' levels of the predictor, the covariance between the dyad members' levels of the outcome, the covariance between the residual error terms of each dyad member, and the fixed effects of each dyad member's predictor onto their own outcome (i.e., actor effects) and onto their partner's outcome (i.e., partner effects).

\section{Results}

\section{Descriptive Statistics}

Descriptive statistics and the number of participants missing data from key study variables are displayed in Table 1. 


\section{Table 1}

Descriptive Statistics

\begin{tabular}{llllll}
\hline Measure & $M$ & $S D$ & Observed Range & $\alpha$ & Missing \\
\hline AWIRS - Overt Violence & 0.27 & 0.45 & $0.00,1.00$ & .80 & 3 \\
IOS & 5.51 & 1.29 & $2.00,7.00$ & - & 4 \\
IPAT - Implicit Partner Value & 111.82 & 455.86 & $-858.88,2343.38$ & .68 & 3 \\
MRIAT - Friend Aggression & 2.41 & 0.77 & $1.00,4.00$ & .89 & 2 \\
MRIAT - Partner Aggression & 2.21 & 0.77 & $1.00,4.00$ & .90 & 2 \\
MRIAT - Stranger Aggression & 2.41 & 0.81 & $1.00,4.00$ & .92 & 2 \\
PRQC - Explicit Partner Value & 6.43 & 0.61 & $3.33,7.00$ & .93 & 3 \\
\hline
\end{tabular}

Note. IPAT = Implicit Partner Attitudes Task; AWIRS = Abuse Within Intimate Relationships Scale; IOS = Inclusion of Other in the Self scale, MRIAT = MRI Aggression Task, PRQC = Perceived Relationship Quality Components inventory.

\section{Aggression Task Behavioral Data}

Across both runs of the MRI aggression task, a repeated-measures ANOVA revealed that noise blast volumes were characterized by a main effect of target, $F(2$, $194)=10.82, p<.001, \omega^{2}=.01$ (Figure 4). According to pairwise contrasts, noise blasts directed at partners were less loud than noise blasts directed at friends, $t(194)=-3.98, p$ $<.001, d=-0.40$, and strangers, $t(194)=-4.08, p<.001, d=-0.41$. Friend and stranger noise blast levels did not differ from one another, $t(194)=-0.10, p=.922, d=-0.01$. 


\section{Figure 4}

Raincloud and Box Plots of Participants' Noise Blast Settings Across the MRI Aggression Task, by Target

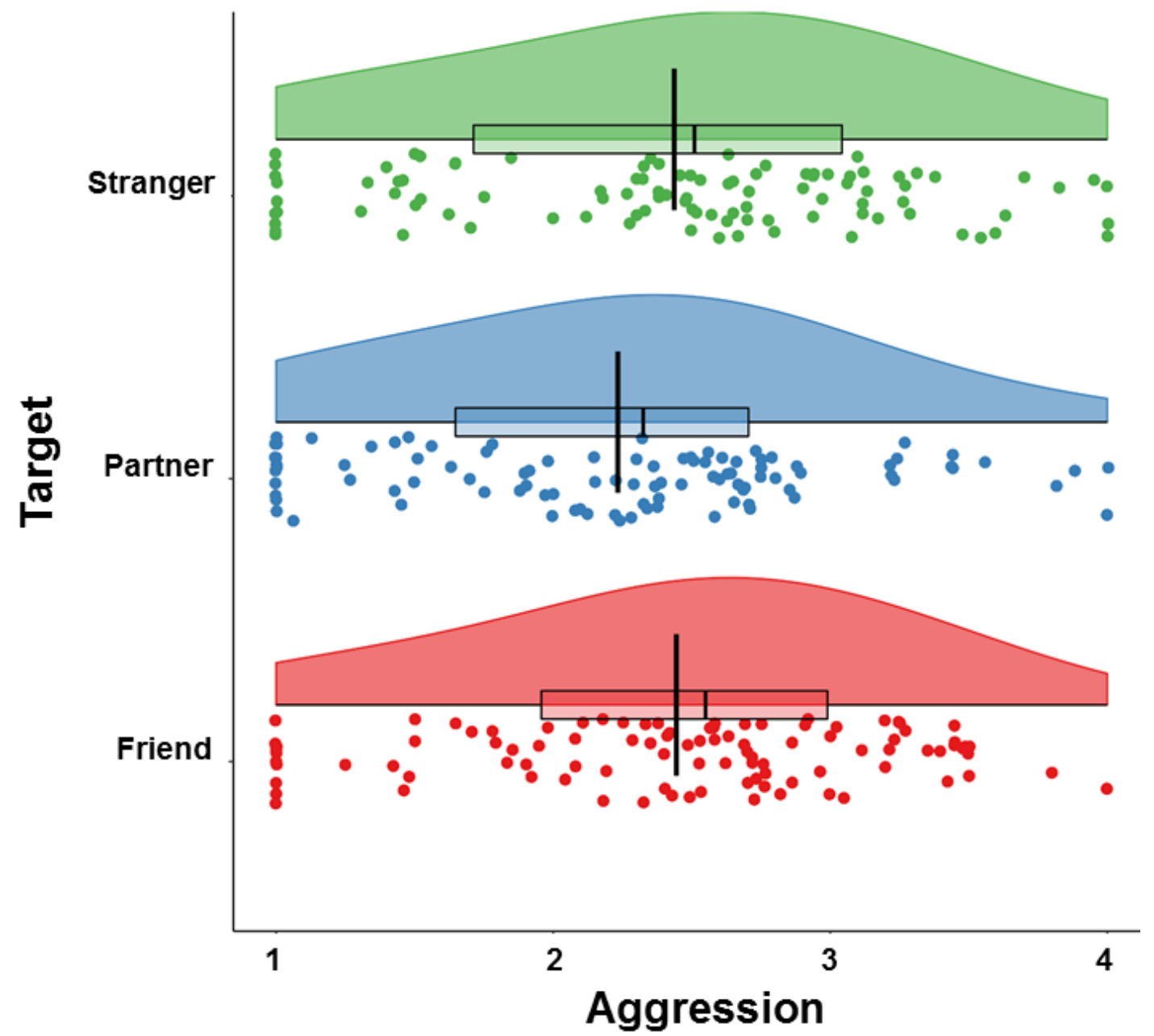

Note. Larger vertical lines indicate means.

\section{Real-World IPA}

As one might expect from a sample not recruited for having substantial life histories of IPA, reports of real-world IPA on the AWIRS' Overt Violence subscale were problematically, positively skewed (skewness $=3.97)$ and zero-inflated $(73.2 \%$ zeroes). To address this issue, we deviated from our preregistration plan and re-coded these scores in a binary fashion such that any endorsement of past IPA was scored as 1 and 
no past IPA was scored as 0 (as in Chester \& DeWall, 2019). A total of $26.8 \%$ of participants reported perpetrating a past act of overt IPA. Supporting the validity of the MRI aggression task, multilevel modeling revealed that noise blast settings for participants' intimate partners were higher among participants who had perpetrated a 'real-world' act of overt intimate partner aggression as compared to non-perpetrators, $\beta$ $=.23(95 \% \mathrm{Cl}=.04, .43), t(91.04)=2.31, p=.023$. This effect was not observed for noise blast settings towards friends or strangers on the aggression task.

\section{Neural Correlates of IPA}

Consistent with our predictions, the louder that participants set noise blasts for their intimate partner during Partner Aggression Decisions (compared to Friend and Stranger Aggression Decisions), the less activity we observed in the VMPFC and neighboring ventrolateral PFC: 396 voxels; Brodmann's areas 10 and 11; peak voxel: $Z$ $=-4.34, \mathrm{MNI}$ coordinates: $\mathrm{x}=-10, \mathrm{y}=66, \mathrm{z}=2$ (Figure 5). 


\section{Figure 5}

Activity in Ventromedial and Ventrolateral Prefrontal Cortices during Partner $>$ NonPartner Aggression Decisions, Which was Negatively Linked to Intimate Partner

\section{Aggression}

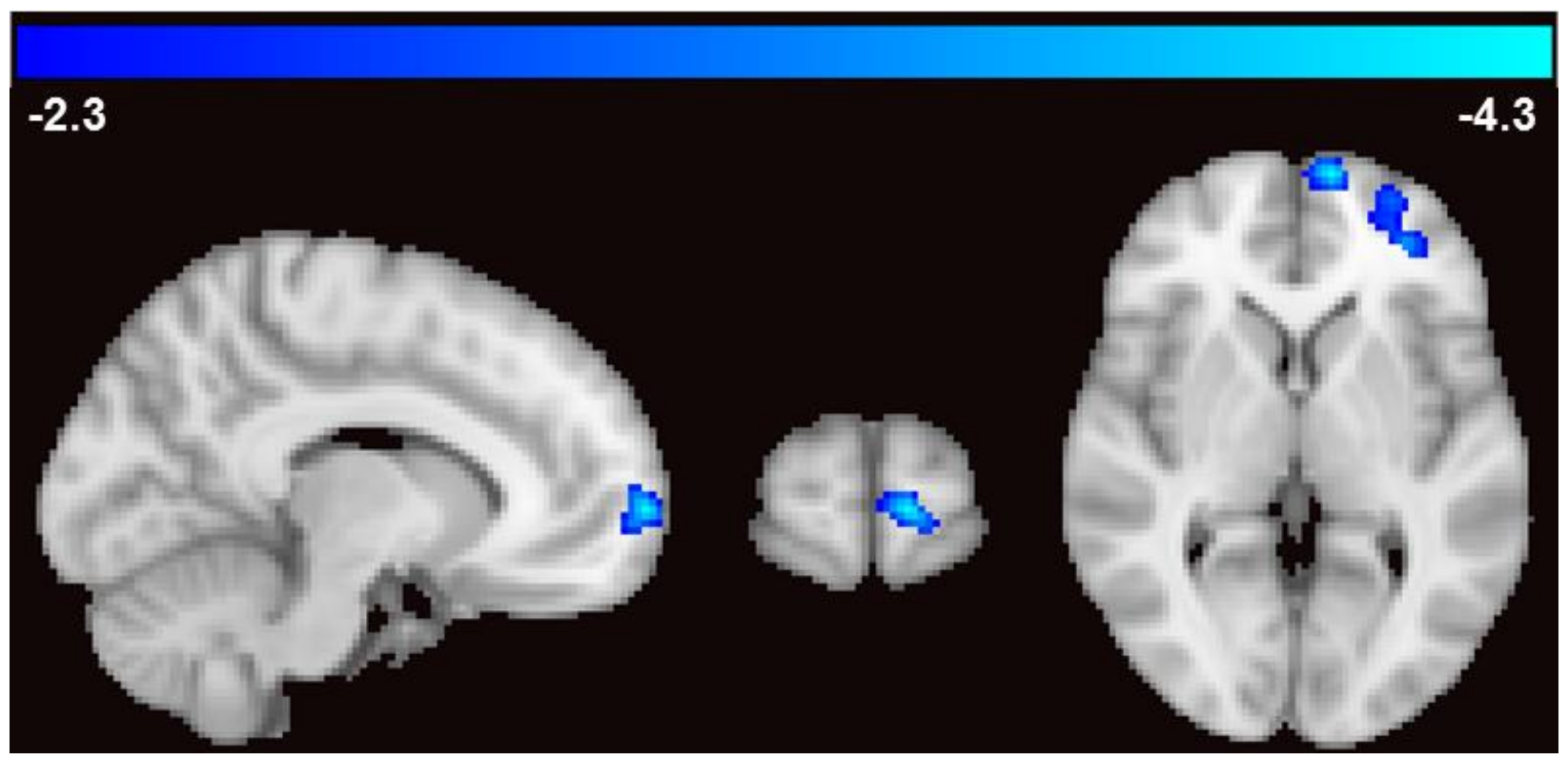

\section{Correlations With Real-World IPA}

Extending these findings outside of the laboratory, multilevel modeling revealed that IPA-related VMPFC activity was also negatively associated with acts of 'real-world' IPA as measured by the AWIRS' Overt Violence subscale, $\beta=-.22(95 \% \mathrm{Cl}=-.42$, $.03), t(85.29)=2.22, p=.029$.

\section{Reverse Inference Correlations}

Self-partner overlap. Using multilevel modeling, IPA-related VMPFC activity was not significantly associated with perceptions of self-partner overlap as measured by the Inclusion of Other in Self Scale, $\beta=.00(95 \% C l=-.21, .19), t(92)=-0.09, p=.927$. Exploratory Bayesian analyses using JASP (v. 0.11.1; JASP Team, 2020) found moderate evidence in favor of the null hypothesis, $B F_{10}=0.13$. 
Explicit partner value. Using multilevel modeling, IPA-related VMPFC activity was unassociated with overall self-reported relationship quality as measured by the total score from the Perceived Relationship Quality Components inventory, $\beta=-.04$ (95\% Cl $=-.24, .17), t(93)=-0.36, p=.723$. Exploratory Bayesian analyses found moderate evidence in favor of the null hypothesis, $B F_{10}=0.14$.

Implicit partner value. Using multilevel modeling, IPA-related VMPFC activity was not associated with implicit partner value as measured by the implicit partner attitudes task, $\beta=.12(95 \% \mathrm{Cl}=-.08, .32), t(95)=1.20, p=.235$. Exploratory Bayesian analyses found only weak evidence in favor of the null hypothesis, $B F_{10}=0.54$. See Supplemental Document 1 for descriptive statistics and more details about the implicit partner attitudes task.

\section{Functional Connectivity With DMPFC}

We next examined if partner aggression was associated with differences not only in VMPFC activity, but in its functional connectivity to more dorsal aspects of the medial PFC. Constraining analyses to the DMPFC (via the medial aspect of the superior frontal gyrus mask from the Automated Anatomical Labeling Atlas; Tzourio-Mazoyer et al., 2002), setting louder noise blasts for intimate partners was positively associated with functional connectivity between the VMPFC seed region and a cluster in the DMPFC during Partner > Non-Partner Aggression Decisions: 116 voxels; Brodmann's area 9; peak voxel: $Z=3.30, \mathrm{MNI}$ coordinates: $\mathrm{x}=2, \mathrm{y}=48, z=46$ (Figure 6). As such, IPA may arise from the DMPFC's functional coupling with the VMPFC, potentially constraining its ability to inhibit IPA. Expanding these connectivity analyses across the entire brain did not reveal any other regions with greater VMPFC connectivity during 
aggression Partner > Non-Partner Aggression Decisions.

\section{Figure 6}

VMPFC-Based Functional Connectivity With Dorsomedial Prefrontal Cortex During Partner > Non-Partner Aggression Decisions, Which was Positively Linked to Intimate Partner Aggression

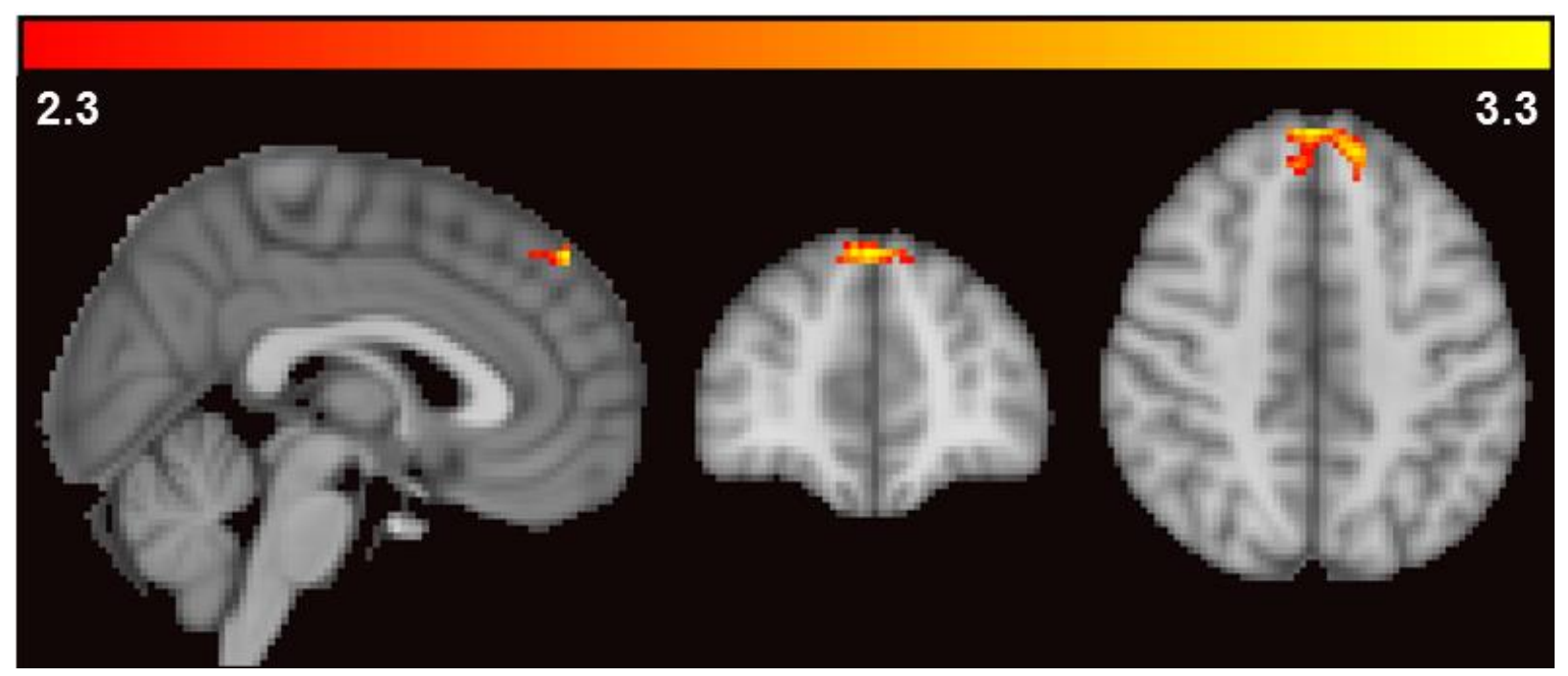

\section{Provocation-Related Neural Correlates of IPA}

Subsequent fMRI analyses examined the whole-brain correlates of partner provocation (compared to provocations from friends and strangers) and their association with IPA. The louder the noise blasts that participants set for their intimate partner during the aggression task, the less activity we observed in the precuneus and posterior cingulate cortex (PCC) during partner provocation: 753 voxels; Brodmann's areas 7, 23, and 31; peak voxel: $Z=-3.75, M N I$ coordinates: $x=-6, y=-58, z=20$ (Figure 7). This provocation-related PCC activity was only associated with partner aggression on the MRI task, $\beta=-0.25, t(96.4)=-2.58, p=.011$, and not any other outcome variables, as estimated by multilevel modeling. 


\section{Figure 7}

Activity in Precuneus and Posterior Cingulate Cortex During Parametrically-Modulated Partner > Non-Partner Provocation Events That was Negatively Linked to Intimate Partner Aggression

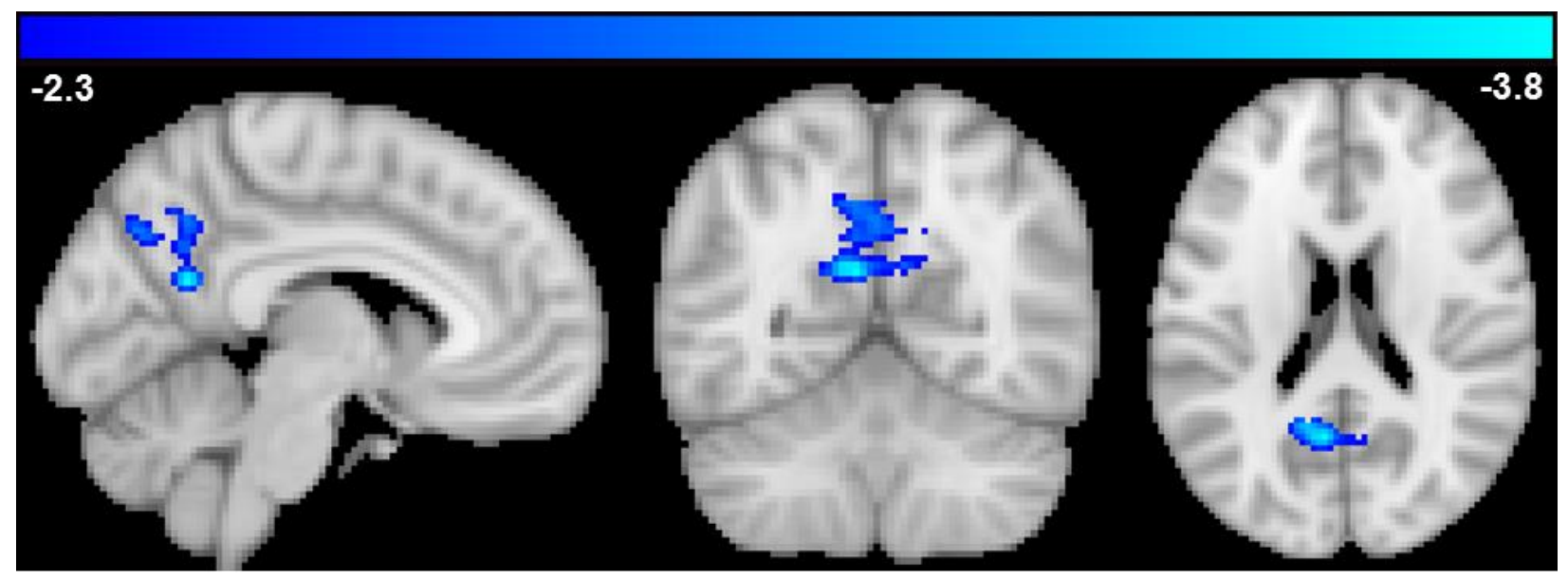

\section{Actor-Partner Independence Modeling}

PCC ---> IPA. To examine whether female and male partners' PCC reactivity to provocation exerted effects on their own and each other's IPA levels on the MRI aggression task, we conducted actor-partner independence modeling (APIM). The model demonstrated an actor effect for linking participants' own PCC activity to lesser IPA for men, but not women (Figure 8). We observed no partner effects. 
Figure 8

Actor-Partner Independence Model, in Which Men's Provocation-Related PCC Activity Predicted Less Intimate Partner Aggression Among Men

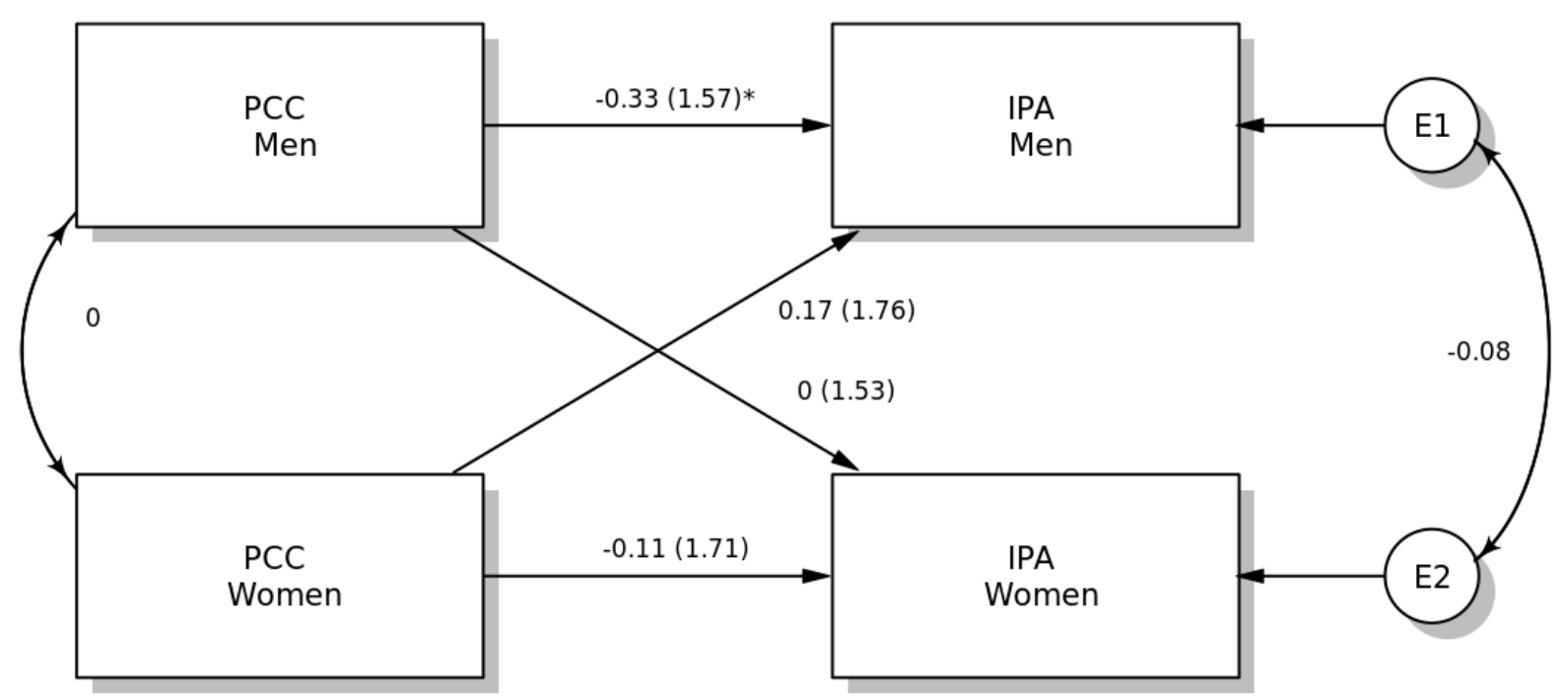

Note. Values represent standardized path coefficients, parenthesized values represent standard errors of path coefficients. ${ }^{*} p<.05,{ }^{* *} p<.01$.

VMPFC ---> IPA. A second APIM examined whether female and male partners' VMPFC activity during IPA decisions exerted effects on their own and each other's IPA levels from the noise blast task. In contrast to the previous model, this APIM revealed that the actor effect linking participants' own VMPFC activity to lesser IPA was specific to women and not observed among men (Figure 9). Further, we observed no partner effects. 
Figure 9

Actor-Partner Independence Model, in Which IPA-Related VMPFC Activity Predicted Less Intimate Partner Aggression Among Women

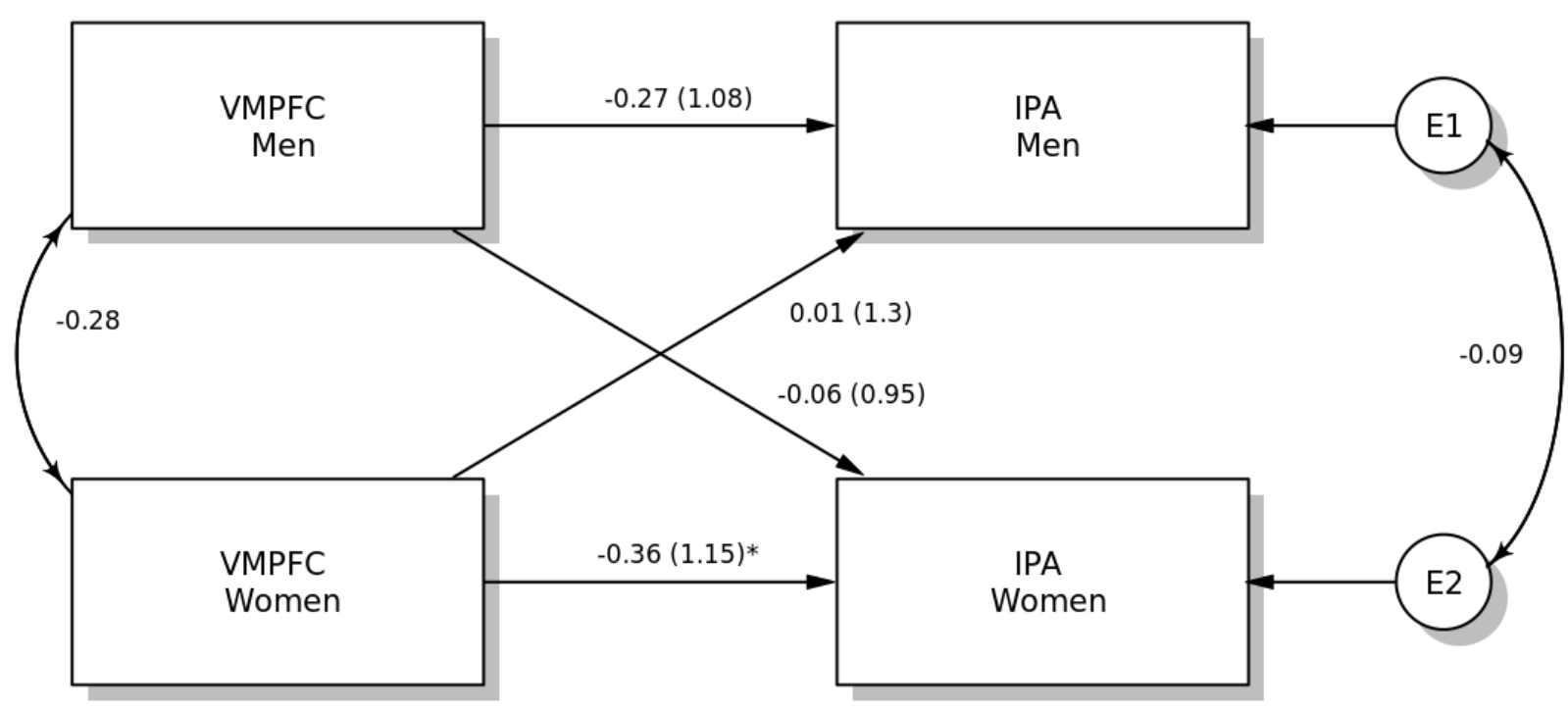

Note. Values represent standardized path coefficients, parenthesized values represent standard errors of path coefficients. ${ }^{*} p<.05$.

PCC ---> VMPFC. A third and final APIM examined whether female and male partners' provocation-related PCC activity exerted effects on their own and each other's VMPFC activity during IPA events. This model replicated the first APIM, in which the PCC actor effect was specific to males (Figure 10). Further, we observed a partner effect, such that males' PCC activity predicted lower subsequent VMPFC activity among women. 


\section{Figure 10}

Actor-Partner Independence Model, in Which Men's Provocation-Related PCC Activity Predicted Greater IPA-Related VMPFC Activity Among Men and Less IPA-Related VMPFC Activity Among Women

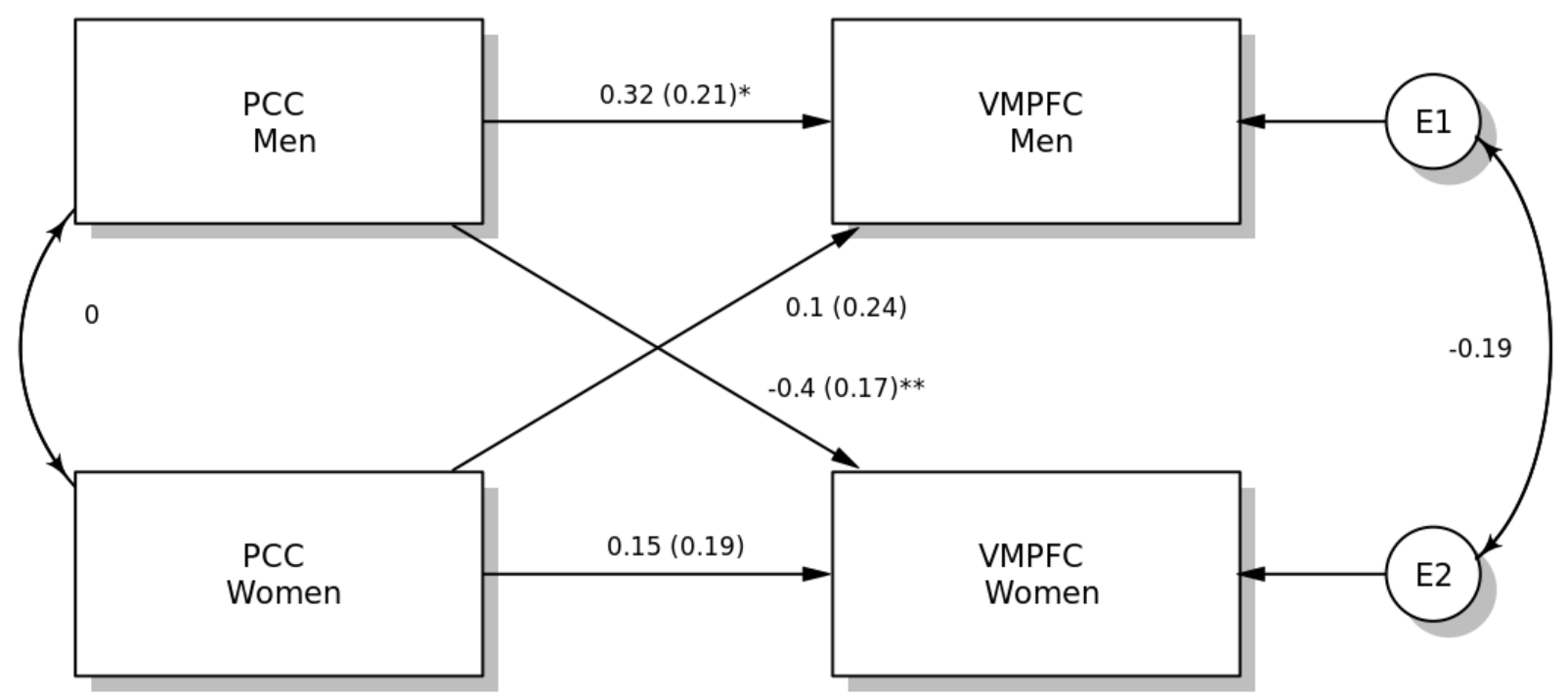

Note. Values represent standardized path coefficients, parenthesized values represent standard errors of path coefficients. ${ }^{*} p<.05,{ }^{* *} p<.01$.

\section{Discussion}

This investigation examined the in vivo neural and psychological processes occurring immediately preceding and during acts of IPA. We found that IPA was linked to altered functioning in midline cortical regions that subserve social-psychological processes. Such a window into these mechanisms provides us with new knowledge about the complex forces driving IPA.

\section{The VMPFC and Reduced IPA}

IPA, as compared to aggression perpetrated against a close friend or a stranger, was negatively associated with concurrent activity in the VMPFC. The use of these two control conditions excluded the confounding effects of general familiarity and the 
different-sex nature of the target of aggression — isolating variance unique to intimate partners. This finding supports a growing literature that identifies the VMPFC as a critical neural constraint on aggression (Beyer et al., 2015; Chester et al., 2017). We extended this literature by showing that the VMPFC is particularly important for reductions in IPA, above-and-beyond other forms of aggressive behavior. Models of IPA are likely to benefit from focusing on the VMPFC, the neural networks it participates in, and the psychological processes it subserves.

\section{Functional Connectivity}

Compared to the negative correlation we observed between IPA VMPFC activity during aggression decisions, we found that IPA was reflected in greater functional coupling between the VMPFC and DMPFC. This finding supports the broader literature on the role of DMPFC function in promoting aggression, especially in the context of IPA (Chester \& DeWall, 2019). The DMPFC serves many functions, such as perspectivetaking (Barrett \& Satpute, 2013) and angry rumination (Denson et al., 2009), yet we were unable to investigate those potential functions here. More research is needed into the DMPFC's psychological functions that promote IPA and how its dynamic interactions with the VMPFC influence this outcome.

\section{Provocation and the PCC}

Participants who were less aggressive towards their partners exhibited greater PCC reactivity to their partners' provocations, which fits with prior work demonstrating that the PCC (in conjunction with the VMPFC) subserves social cognition (Barrett \& Satpute, 2013) and value computation (Liu et al., 2011). This PCC finding meshes well with our broader pattern of results, suggesting that midline cortical regions in the default 
mode network are critical neural substrates of IPA.

\section{Broader Implications for the Psychology and Neuroscience of IPA Perpetration}

Our findings, taken together, suggest several surprising revisions to our initial conceptual model that have important implications for both the psychology and neuroscience of IPA perpetration (original mode: Figure 1; revised model: Figure 11).

\section{Figure 11}

\section{Revised Conceptual Model}

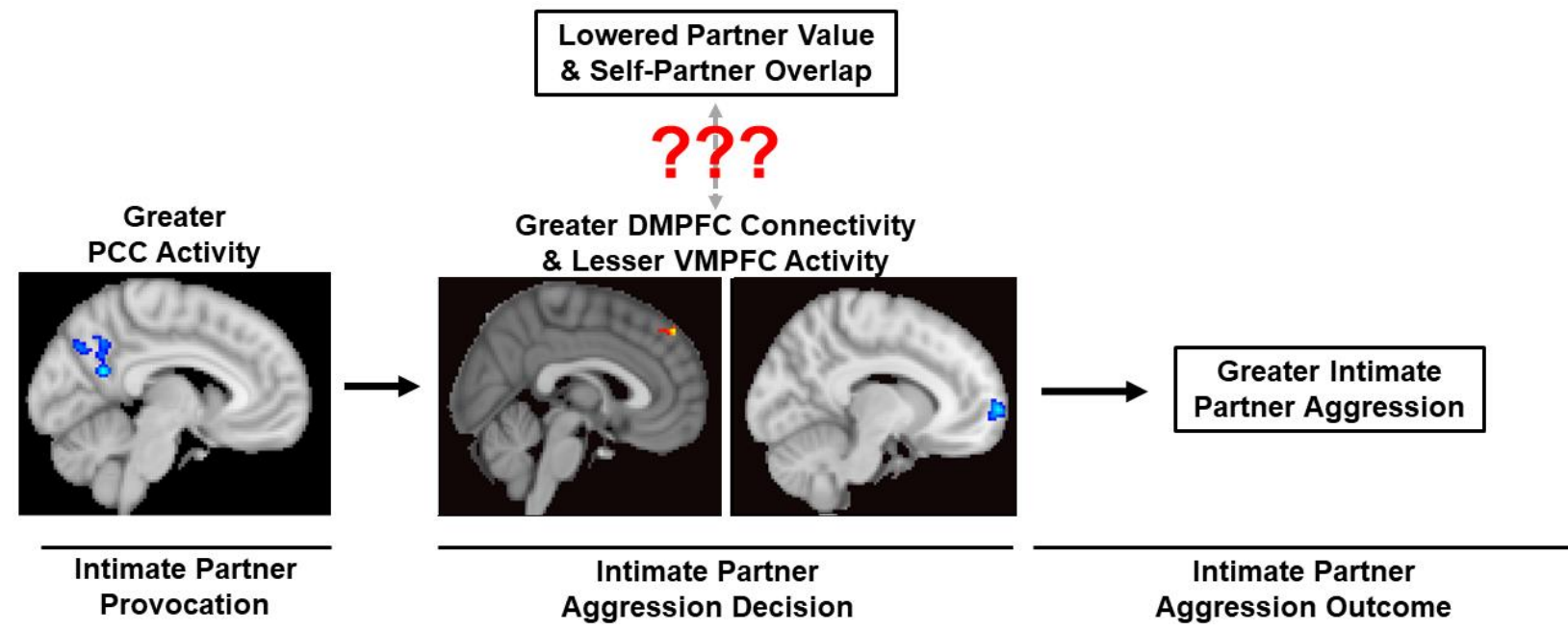

Midline paradigm shift. First, our findings with the PCC point to the importance of neural reactivity to provocation to both subsequent neural activity during aggression decisions and subsequently, to IPA perpetration itself. Little work to date has directly examined IPA's relation to neural reactivity to partner provocation and suggest that the field needs to attend more to provocation reactivity than it has in the past. Further, these PCC findings, in concert with other findings with the DMPFC and VMPFC, strongly support past researchers' focus on these midline cortical regions (Beyer et al., 2015; Chester et al., 2017). This 'midline paradigm shift' is a critical challenge to psychological and neuroscientific approaches to IPA perpetration that focus on impairment in 
executive functions that arise from the dorsolateral aspects of prefrontal and parietal cortices (e.g., Corvo, 2014). A host of criminological and psychological research has suggested that violence perpetration begins where self-control stops (e.g., the General Theory of Crime: Gottfredson \& Hirschi, 1990; self-control models of aggression: Denson, DeWall, \& Finkel, 2012). Though these midline structures that we identified in our study are important for self-regulation, they are not directly implicated in the effortful inhibition of violent impulses that is ascribed to lateral cortical regions. As such, our findings should guide researchers to focus less on effortful self-control and the dorsolateral cortex and more on the alternative self-regulatory processes subserved by midline cortical regions.

Uncertainty around reverse inferences. Second, we were unable to identify self-partner overlap and partner value as psychological correlates of VMPFC activity during aggression decisions. We had a strong evidentiary basis to expect that aggression-related VMPFC activity would reflect the extent to which participants exhibited identity overlap with their partner and with their implicit valuations of their partner (Denny et al., 2012; Lieberman et al., 2019). However, measures of self-partner overlap, as well as implicitly assessed and explicitly assessed partner value were all uncorrelated with aggression-related VMPFC activity. It is possible that self-other overlap was not an ideal candidate for this role because this psychological process is a double-edged sword (i.e., it can promote both benevolence and malevolence in the context of relationships; Galinsky et al., 2005). It is also possible that our moderate sample limited our ability to detect what are real but small effects. Further, the measures of our psychological candidates may have imperfectly captured the intended 
construct, rendering these correlates invalid.

Bayesian analyses revealed moderate evidence for the null hypothesis concerning explicit partner value and self-other overlap. It may be that such self-reports failed to capture more subtle, implicit processes subserved by the VMPFC. Only weak evidence for the null hypothesis was found for the implicit partner value correlations with VMPFC activity. Such weak evidence renders this null result somewhat equivocal and uncertain as to its meaning. Taken together, our results do not fully suggest that these psychological reverse inference variables were or were not valid candidates. While this empirical ambiguity remains, we cannot recommend any revisions to existing neuroscientific models of IPA perpetration and the psychological processes that are theorized to be represented by such biological mechanisms. However, our lack of evidence one way or another allows us to make several recommendations for future research to clear away such ambiguities.

These three null effects leave the interpretation of what psychological processes were being represented by VMPFC activity during aggression open to speculation. Future research might examine other psychological mechanisms that relate to the neural correlates we observed, such as impaired self-regulation and heightened emotional impulsivity — both of which are potent predictors of IPA (Derefinko et al., 2011; Finkel \& Hall, 2018) and correlated with altered VMPFC functioning (Chester et al., 2019; Cyders et al., 2014). Future research might replicate our procedure alongside validated measures of self-regulatory functioning and emotional impulsivity or might systematically manipulate these constructs to examine their role.

\section{Gender and Partner Effects}


When we employed actor-partner independence modeling to separately examine men's and women's interactive effects, we observed that the link between VMPFC's actor effect on IPA was observed only among women and the PCC actor effect was observed only among men. Though these gender differences were themselves not significant, these findings raise the possibility that women's IPA may be influenced more by neural functioning during the aggressive decision (i.e., determining how aggressive to be in the face of provocation) and men's IPA may arise earlier in antagonistic interactions, during acts of provocations themselves. The former possibility would make sense within the context of the larger literature pointing to the predominant role of selfdefense motives among women during acts of IPA, which occur during the aggressive event itself (Stuart, Moore, Hellmuth, Ramsey, \& Kahler, 2006). The latter possibility fits with meta-analytic evidence finding that male perpetrators of IPA exhibit exaggerated levels of angry reactivity to provocation (Norlander \& Eckhardt, 2005). Future research is needed to examine the role of such motives and affective experiences within the neural mechanisms underlying IPA perpetration.

Men's provocation-related PCC reactivity predicted more VMPFC activity during subsequent opportunities for IPA. This result speaks to the possibility that these midline cortical regions dynamically interact with one another to recruit self-regulatory resources during an aggressive interaction. Given that the functional connectivity between such default mode network hubs is linked to angry and aggressive tendencies (Weathersby, King, Fox, Loret, \& Anderson, 2019), there is good reason to expect that this correlation reflects actual self-regulatory connections with implications for IPA perpetration. Yet why this effect was observed only among men remains uncertain. Future research may 
benefit from further exploring this gender difference.

These actor effects were accompanied by a partner effect, such that men's PCC reactivity during provocation predicted lower VMPFC activity during IPA among women. It may be that men's neural responses to provocation are conveyed to their female partners through a yet-unknown mechanism, and that this mechanism may serve to attenuate their self-regulatory neural function during acts of IPA. Thus, women's selfregulation of IPA in the real world may be, in part, a function of how their male partners react to perceived provocation. This conceptualization of these findings would mesh well with the crucial role of self-defense motives for female IPA perpetration (Stuart et al., 2006), as such motives are activated by perceived threatening responses (i.e., angry reactions to perceived provocation). More research that systematically addresses such self-defense motives is needed.

\section{Limitations and Future Directions}

Due to practical and ethical factors, our MRI aggression task was unable to truly simulate the severity and contextual features of naturally occurring IPA. Future research using more ecologically valid aggression measures could examine whether our findings replicate in more naturalistic environments. We also did not explicitly recruit individuals who were particularly prone to IPA. Assuming that appropriate ethical procedures are established, future work should test whether our effects replicate in populations at-risk for IPA perpetration and victimization. It is also worth noting that our findings were often correlational and largely cross-sectional, which could be remedied by experimental or longitudinal designs. Finally, couples of other gender identities, sexual orientations, and exclusivity statuses should be included in future research on this topic. 


\section{Conclusions}

Why do people harm those they love? Answering this question is an urgent errand. Our findings suggest that IPA arises from a complex and dynamic pattern of cortical midline activity during both provocation and aggression and exhibits both actor and partner effects. The psychological processes reflected in this array of neural activity remain unclear, but the established roles of these brain regions in intimacy and value suggest that these are crucial areas to continue to investigate. We hope that future work will harness these mechanisms to reduce harm and promote harmony between intimate partners. 


\section{Funding}

This research was supported by the National Institute on Alcohol Abuse and Alcoholism (NIAAA) of the National Institutes of Health under award number K01AA026647 (PI: Chester). 


\section{Financial Disclosures}

The authors report no financial interests or potential conflicts of interest. 


\section{References}

Allen, J. J., \& Anderson, C. (2017). Aggression and violence: Definitions and distinctions. In P. Sturmey (Ed.), The Wiley handbook of violence and aggression. John Wiley \& Sons.

Aron, A., Aron, E., \& Smollan, D. (1992). Inclusion of Other in the Self Scale and the structure of interpersonal closeness. Journal of Personality and Social Psychology, 63, 596-612.

Aron, A., Aron, E., Tudor, M., \& Nelson, G. (1991). Close relationships as including other in the self. Journal of Personality and Social Psychology, 60, 241-253.

Barrett, L. F. \& Satpute, A. B. (2013). Large-scale brain networks in affective and social neuroscience: towards an integrative functional architecture of the brain. Current Opinion in Neurobiology, 23, 361-372.

Bates, D., Mächler, M., Bolker, B., \& Walker, S. (2014). Fitting linear mixed-effects models using Ime4. Journal of Statistical Software, 67(1), 1-48.

Bell, K. M. \& Naugle, A. E. (2007). Effects of social desirability on students' selfreporting of partner abuse perpetration and victimization. Violence and Victims, 22, 243-256.

Beyer, F., Münte, T. F., Göttlich, M., \& Krämer, U. M. (2015). Orbitofrontal cortex reactivity to angry facial expression in a social interaction correlates with aggressive behavior. Cerebral Cortex, 25(9), 3057-3063.

Black, M.C., Basile, K.C., Breiding, M.J., Smith, S.G., Walters, M.L., Merrick, M.T., Chen, J., \& Stevens, M.R. (2011). The national intimate partner and sexual violence survey (NISVS): 2010 summary report. Atlanta (GA): National Center for 
Injury Prevention and Control, Centers for Disease Control and Prevention.

Retrieved from: http://www.cdc.gov/violenceprevention/pdf/nisvs_report2010a.pdf

Borjesson, W. I., Aarons, G. A., \& Dunn, M. E. (2003). Development and confirmatory factor analysis of the abuse within intimate relationships scale. Journal of Interpersonal Violence, 18, 295-309.

Buades-Rotger, M., Beyer, F., \& Krämer, U. M. (2017). Avoidant responses to interpersonal provocation are associated with increased amygdala and decreased mentalizing network activity. ENeuro, 4(3), ENEURO-0337.

Bueso-Izquierdo, N., Verdejo-Román, J., Contreras-Rodríguez, O., Carmona-Perera, M., Pérez-García, M., \& Hidalgo-Ruzzante, N. (2016). Are batterers different from other criminals? An fMRI study. Social Cognitive and Affective Neuroscience, 11, 852-862.

Capaldi, D. M., Knoble, N. B., Shortt, J. W., \& Kim, H. K. (2012). A systematic review of risk factors for intimate partner violence. Partner Abuse, 3, 231-280.

Chavez, R. S., Heatherton, T. F., \& Wagner, D. D. (2016). Neural population decoding reveals the intrinsic positivity of the self. Cerebral Cortex, 27, 5222-5229.

Chester, D. S., Bell, S. B., DeWall, C. N., West, S. J., Romero-Lopez, M., \& Craig, A. W. (2019). Neural correlates of intertemporal choice in aggressive behavior. Aggressive Behavior, 45(5), 507-516.

Chester, D. S. \& DeWall, C. N. (2016). The pleasure of revenge: Retaliatory aggression arises from a neural imbalance toward reward. Social Cognitive and Affective Neuroscience, 11, 1173-1182. 
Chester, D. S. \& DeWall, C. N. (2017). The roots of intimate partner violence. Current Opinion in Psychology, 19, 55-59.

Chester, D. S. \& DeWall, C. N. (2019). Intimate partner violence perpetration corresponds to a dorsal-ventral gradient in medial PFC reactivity to interpersonal provocation. Social Neuroscience, 14, 173-182.

Chester, D. S. \& Lasko, E.N. (2019). Validating a standardized approach to the Taylor Aggression Paradigm. Social Psychological and Personality Science, 10(5), 620631.

Chester, D. S., Lynam, D. R., Milich, R., \& DeWall, C. N. (2017). Physical aggressiveness and gray matter deficits in ventromedial prefrontal cortex. Cortex, 97, 17-22.

Corvo, K. (2014). The role of executive function deficits in domestic violence perpetration. Partner Abuse, 5(3), 342-356.

Cyders, M. A., Dzemidzic, M., Eiler, W. J., Coskunpinar, A., Karyadi, K., \& Kareken, D. A. (2014). Negative urgency and ventromedial prefrontal cortex responses to alcohol cues: fMRI evidence of emotion-based impulsivity. Alcoholism: Clinical and Experimental Research, 38(2), 409-417.

Denny, B. T., Kober, H., Wager, T. D., \& Ochsner, K. N. (2012). A meta-analysis of functional neuroimaging studies of self- and other judgments reveals a spatial gradient for mentalizing in medial prefrontal cortex. Journal of Cognitive Neuroscience, 24, 1742-1752.

Denson, T. F., DeWall, C. N., \& Finkel, E. J. (2012). Self-control and aggression. Current Directions in Psychological Science, 21(1), 20-25. 
Denson, T. F., Pedersen, W. C., Ronquillo, J., \& Nandy, A. S. (2009). The angry brain: Neural correlates of anger, angry rumination, and aggressive personality. Journal of Cognitive Neuroscience, 21, 734-744.

Derefinko, K., DeWall, C. N., Metze, A. V., Walsh, E. C., \& Lynam, D. R. (2011). Do different facets of impulsivity predict different types of aggression?. Aggressive Behavior, 37(3), 223-233.

Fazio, R. H. (2007). Attitudes as object-evaluation associations of varying strength. Social Cognition, 25, 603-637.

Figueredo, A., Jacobs, W., Gladden, P., Bianchi, J., Patch, E., Kavanagh, P., ... \& Li, N. (2018). Intimate partner violence, interpersonal aggression, and life history strategy. Evolutionary Behavioral Sciences, 1, 1-31.

Finkel, E. J., DeWall, C. N., Slotter, E. B., McNulty, J. K., Pond, R. S., \& Atkins, D. C. (2012). Using I3 theory to clarify when dispositional aggressiveness predicts intimate partner violence perpetration. Journal of Personality and Social Psychology, 102, 533-549.

Finkel, E. J., Eastwick, P. W., \& Reis, H. T. (2015). Best research practices in psychology: Illustrating epistemological and pragmatic considerations with the case of relationship science. Journal of Personality and Social Psychology, 108, 275-297.

Finkel, E. J., \& Hall, A. N. (2018). The I3 model: A metatheoretical framework for understanding aggression. Current Opinion in Psychology, 19, 125-130.

Flanagan, J. C., Yonce, S., Calhoun, C. D., Back, S. E., Brady, K. T., \& Joseph, J. E. (2019). Preliminary development of a neuroimaging paradigm to examine neural 
correlates of relationship conflict. Psychiatry Research: Neuroimaging, 283, 125134.

Fletcher, G. J., Simpson, J. A., \& Thomas, G. (2000). The measurement of perceived relationship quality components: A confirmatory factor analytic approach. Personality and Social Psychology Bulletin, 26(3), 340-354.

Galinsky, A. D., Ku, G., \& Wang, C. S. (2005). Perspective-taking and self-other overlap: Fostering social bonds and facilitating social coordination. Group Processes \& Intergroup Relations, 8, 109-124.

Giancola, P. R. \& Parrott, D. J. (2008). Further evidence for the validity of the Taylor aggression paradigm. Aggressive Behavior, 34, 214-229.

Gottfredson, M. R., \& Hirschi, T. (1990). A general theory of crime. Stanford University Press.

Halmos, M. B., Parrott, D. J., Henrich, C. C., \& Eckhardt, C. I. (2020). The structure of aggression in conflict-prone couples: Validation of a measure of the Forms and Functions of Intimate Partner Aggression (FFIPA). Psychological Assessment, 32(5), 461-472.

Heller, R., Stanley, D., Yekutieli, D., Rubin, N., \& Benjamini, Y. (2006). Cluster-based analysis of fMRI data. Neurolmage, 33, 599-608.

Hughes, B. L. \& Beer, J. S. (2012). Orbitofrontal cortex and anterior cingulate cortex are modulated by motivated social cognition. Cerebral Cortex, 22, 1372-1381.

JASP Team (2020). JASP (version 0.11.1) [Software]. Available from https://jaspstats.org/.

Kawamichi, H., Sasaki, A. T., Matsunaga, M., Yoshihara, K., Takahashi, H. K., Tanabe, 
H. C., \& Sadato, N. (2013). Medial prefrontal cortex activation is commonly invoked by reputation of self and romantic partners. PLOS ONE, 8, e74958.

Kelley, H. H. \& Thibaut, J. W. (1978). Interpersonal relations: A theory of interdependence. John Wiley \& Sons.

King, A. R., \& Russell, T. D. (2019). Lifetime Acts of Violence Assessment (LAVA) predictors of laboratory aggression. Aggressive behavior, 45(5), 477-488.

Krämer, U. M., Jansma, H., Tempelmann, C., \& Münte, T. F. (2007). Tit-for-tat: The neural basis of reactive aggression. Neurolmage, 38, 203-211.

Kuznetsova, A., Brockhoff, P. B., \& Christensen, R. H. (2017). ImerTest package: Tests in linear mixed effects models. Journal of Statistical Software, 82(13), 1-26.

LeBel, E. P. \& Campbell, L. (2009). Implicit partner affect, relationship satisfaction, and the prediction of romantic breakup. Journal of Experimental Social Psychology, 45, 1291-1294.

Lieberman, M. D., Straccia, M. A., Meyer, M. L., Du, M., \& Tan, K. M. (2019). Social, self, (situational), and affective processes in medial prefrontal cortex (MPFC): Causal, multivariate, and reverse inference evidence. Neuroscience \& Biobehavioral Reviews, 99, 311-328.

Liu, X., Hairston, J., Schrier, M., \& Fan, J. (2011). Common and distinct networks underlying reward valence and processing stages: A meta-analysis of functional neuroimaging studies. Neuroscience \& Biobehavioral Reviews, 35, 1219-1236.

Lotze, M., Veit, R., Anders, S., \& Birbaumer, N. (2007). Evidence for a different role of the ventral and dorsal medial prefrontal cortex for social reactive aggression: An interactive fMRI study. Neurolmage, 34, 470-478. 
Marín-Morales, A., Bueso-Izquierdo, N., Hidalgo-Ruzzante, N., Pérez-García, M., Catena-Martínez, A., \& Verdejo-Román, J. (2020). "Would you allow your wife to dress in a miniskirt to the party"? Batterers do not activate default mode network during moral decisions about intimate partner violence. Journal of Interpersonal Violence, 886260520926494.

McNulty, J. K., Olson, M. A., Meltzer, A. L., \& Shaffer, M. J. (2013). Though they may be unaware, newlyweds implicitly know whether their marriage will be satisfying. Science, 342, 1119-1120.

Mitchell, J. P., Macrae, C. N., \& Banaji, M. R. (2006). Dissociable medial prefrontal contributions to judgments of similar and dissimilar others. Neuron, 50, 655- 663.

Murray, S. L. (1999). The quest for conviction: Motivated cognition in romantic relationships. Psychological Inquiry, 10, 23-34.

Norlander, B., \& Eckhardt, C. (2005). Anger, hostility, and male perpetrators of intimate partner violence: A meta-analytic review. Clinical Psychology Review, 25(2), 119152.

Parrott, D., Miller, C., \& Hudepohl, A. (2015). Immediate and short-term reactions to participation in laboratory aggression research. Psychology of Violence, 5, 209216.

R Core Team (2020). R: A language and environment for statistical computing (version 3.6) [Software]. Available from https://www.R-project.org/.

Repple, J., Pawliczek, C. M., Voss, B., Siegel, S., Schneider, F., Kohn, N., \& Habel, U. (2017). From provocation to aggression: The neural network. BMC Neuroscience, 18(1), 1-9. 
Richard, F. D., Bond Jr, C. F., \& Stokes-Zoota, J. J. (2003). One hundred years of social psychology quantitatively described. Review of General Psychology, 7, 331-363.

Smith, S. M., Jenkinson, M., Woolrich, M. W., Beckmann, C. F., Behrens, T. E. J., Johansen-Berg, H., ... Matthews, P. M. (2004). Advances in functional and structural MR image analysis and implementation as FSL. Neurolmage, 23, S208-S219.

Stas, L, Kenny, D. A., Mayer, A., \& Loeys, T. (2018). Giving dyadic data analysis away: A user-friendly app for actor-partner interdependence models. Personal Relationships, 25 (1), 103-119.

Stuart, G. L., Moore, T. M., Hellmuth, J. C., Ramsey, S. E., \& Kahler, C. W. (2006). Reasons for intimate partner violence perpetration among arrested women. Violence Against Women, 12(7), 609-621.

Thornton, A. J., Graham-Kevan, N., \& Archer, J. (2013). Development and confirmatory factor analysis of the non-violent and violent offending behavior scale (NVOBS). Aggressive Behavior, 39(3), 171-181.

Tzourio-Mazoyer, N., Landeau, B., Papathanassiou, D., Crivello, F., Etard, O., Delcroix, N., ... \& Joliot, M. (2002). Automated anatomical labeling of activations in SPM using a macroscopic anatomical parcellation of the MNI MRI single-subject brain. Neurolmage, 15, 273-289.

Verdejo-Román, J., Bueso-Izquierdo, N., Daugherty, J. C., Pérez-García, M., \& HidalgoRuzzante, N. (2019). Structural brain differences in emotional processing and regulation areas between male batterers and other criminals: A preliminary study. Social Neuroscience, 14(4), 390-397. 
Weathersby, F. L., King, J. B., Fox, J. C., Loret, A., \& Anderson, J. S. (2019). Functional connectivity of emotional well-being: Overconnectivity between default and attentional networks is associated with attitudes of anger and aggression. Psychiatry Research: Neuroimaging, 291, 52-62. 


\section{Supplemental Document 1}

\section{Implicitly Partner Attitudes Task}

Participants categorized words with impressive accuracy, $M=95.68 \%$ correct, $S D=5.56 \%$, range: $75.00-100.00 \%$, suggesting that they took the implicit partner attitudes task seriously. Incorrect responses were excluded from subsequent analyses. Response times were characterized by non-significant main effects of prime-type and word-type, which were qualified a significant word-type by prime-type interaction (Supplemental Figure 1; Supplemental Table 1). Simple slopes contrasts revealed that participants were faster at identifying positive than negative words after partner primes, $t(190.81)=-2.59, p=.010$, and this effect was reversed after stranger primes, $t(190.81)$ $=5.09, p<.001$.

\section{Supplemental Figure 1}

Mean Response Times (and 95\% Cls) for the Implicit Partner Attitudes Task as a Function of Word-Type and Prime-Type

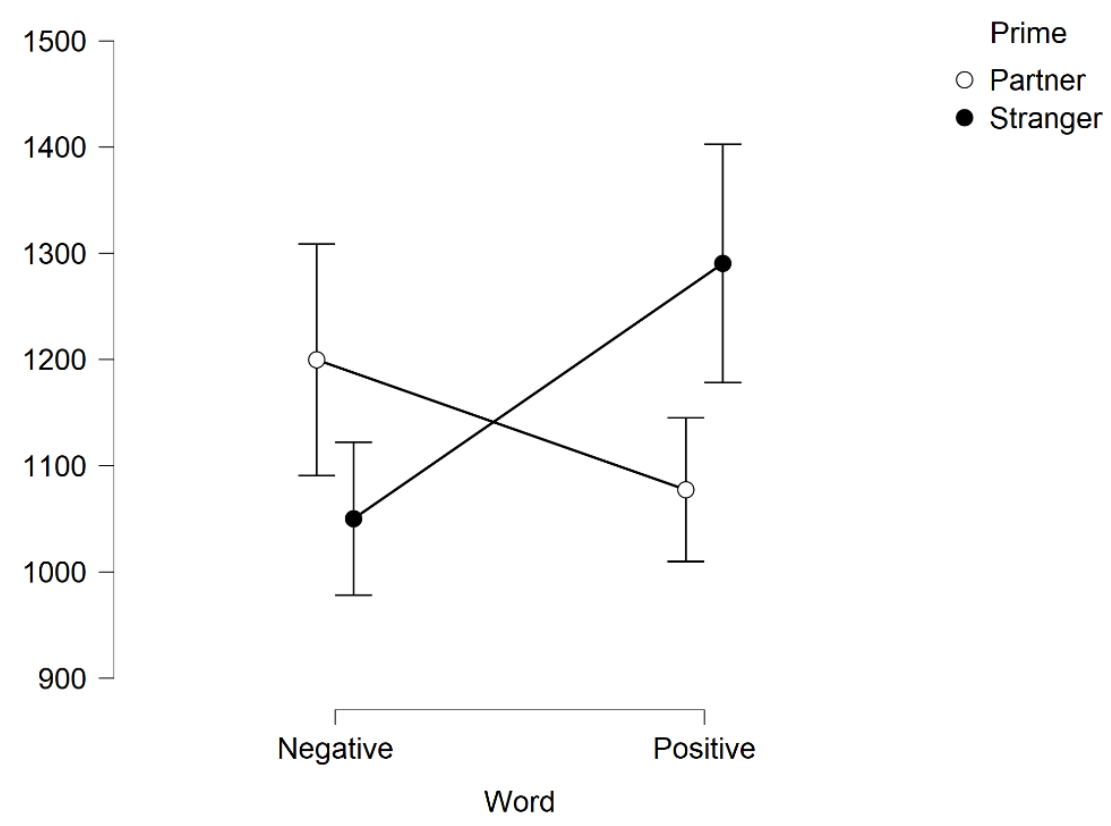




\section{Supplemental Table 1}

Summary of Effects on IPAT Response Times

\begin{tabular}{llll}
\hline Effect & $F(1,98)$ & $p$ & $\omega^{2}$ \\
\hline Prime-Type & 3.73 & .056 & .00 \\
Word-Type & 1.21 & .274 & .00 \\
Prime x Word & 25.29 & $<.001$ & .04
\end{tabular}

Note. AWIRS = Abuse Within Intimate Relationships Scale, IOS = Inclusion of Other in the Self Scale, IPAT = Implicit Partner Attitudes Task, MRIAT = MRI Aggression Task. 